\title{
SIGNIFICACIÓN, ASPECTOS Y VALORES DE LAS ORACIONES CONDICIONALES*
}

ANa-Jimena DeZa EnRfQuez

UNED

\section{INTRODUCCIÓN}

No abundan hoy en día los estudios pormenorizados sobre las condicionales. La cuestión de la condicionalidad se torna compleja porque exige ser tratada desde la filosofía, en razón de los factores lógicos que comprende; desde la psicología, por los múltiples matices que encierra; y, por supuesto, desde la más correcta lingüística. El problema en el ámbito de la condicionalidad surge cuando la relación entre un hecho y otro que es circunstancia de aquél puede formularse de distinta manera, mediante nexos y estructuras temporales, causales, condicionales, adversativos, etc., etc.; pero eso no quiere decir que la formulación temporal implique condicionalidad, ni viceversa: Cuando vengas, pondremos un disco no es sustituible por «si vienes» (puedo tener seguridad de que vendrás), ni «si vienes» por «cuando vengas», que rechaza el

* El presente artículo fue, en origen, un trabajo sobre 350 casos de oraciones condicionales. Por razones de espacio, resulta evidente que hubo de ser podado en la teoría y en la práctica, siendo obvio que las conclusiones deducidas en aquél difieren de las que aquí ofrecemos, aunque esto no afecta a lo fundamental. 
carácter hipotético. Lo mismo cabe decir de las equivalencias entre condicionales y causales, finales, consecutivas, etc. Hay, sí, casos especiales en que cuando no introduce subordinada temporal, sino condicional ( $Y$ cuando así sea, nos resignaremos = "y si así no fuere", "y si así no es»); y también construcciones de forma condicional con si cuyo contenido es enunciativo, causal, adversativo, temporal, etc.

Nos enfrentamos, por tanto, a esta problemática, no sólo desde el punto de vista nocional, de demarcación estricta de la condicionalidad, su doble plano formal y semántico y las partículas que la anuncian, sino que $-\mathrm{y}$ mucho más decisiva - es la delimitación de las distintas clases de oraciones condicionales, si nos atenemos a los cruces con otras adverbiales, y, sobre todo, a la modalidad que las condicionales presentan en función de los tiempos verbales que rigen y que juegan un papel fundamental que afecta a su esencialidad más intrínseca.

\section{NOCIÓN DE ORACIÓN CONDICIONAL}

Las definiciones que dan los gramáticos sobre el período condicional son muy próximas y casi todas ellas coincidentes. El Esbozo de la Real Academia sostiene que uel período condicional, llamado también hipotético, consta de dos oraciones relacionadas mediante la conjunción si. Una de ellas, la que expresa la condición, es la subordinada y se llama hipótesis, y más comúnmente, prótasis. La principal enuncia el resultado o consecuencia y recibe el nombre de apódosis» ${ }^{1}$. En la línea de la Academia y con anterioridad a ella, se encuentra la noción de condicional de Andrés Bello, quien afirma: «llamaremos hipótesis aquel miembro de la oración que la significa y apódosis el otro miembro que significa el efecto o consecuencia de la condición» ${ }^{2}$. Cejador ${ }^{3}$ especifica algo más los términos de las condicionales, ya que para él «la principal o subordinante es la apódosis, o consiguiente o condicionado, que pende

${ }^{1}$ Real Academia Española, Esbozo de una nueva gramática de la lengua española, Madrid, Espasa Calpe, 1." edición: 1973; duodécima reimpresión: 1989, p. 554.

2 A. Bello, Gramática de la lengua castellana, destinada al uso de los americanos. Con notas de Rufino José Cuervo, Buenos Aires, Sopena, 1.' edición: 1945, p. 613. Recientemente debemos una edición y estudio a Ramón Trujillo en Arco Libros, Madrid, 1988, 2 vols.

3 Julio Cejador y Frauca, La lengua de Cervantes, t. I, Madrid, Jaime Ratés, 1905, p. 260. 
de la condición expresada por la secundaria o subordinada, o prótasis, o hipótesis o antecedente».

Rafael $\mathrm{Seco}^{4}$ y Marcos Marín 5 no aportan nada distinto a la ya conocida definición de oración condicional. Por su parte, Gili Gaya sólo añade que «con ellas hacemos depender el cumplimiento de lo enunciado en la oración principal de la realización de la oración subordinada» 6 .

Lo expuesto hasta ahora se reduce, pues, al esquema siguiente:

O. subordinada $=$ hipótesis o prótasis $=$ antecedente $\mathrm{o}$ condición

O. principal (o. subordinante) $=$ apódosis $=$ consiguiente o condición

Hasta aquí la opinión, muy afín, de los gramáticos más tradicionales sobre la oración condicional. Todos ellos coinciden, además, en la caracterización de éstas como subordinadas adverbiales; todos, excepto Marcos Marín 7 , permanecen fieles, por otra parte, a la tradicional terminología de hipótesis y prótesis, aunque incluya insuficiencias obvias. Olvidan, sin embargo, algo muy importante: el tipo de relación entre prótasis y apódosis, así como la evidente diferencia que entraña, desde el punto de vista filosófico, la pareja de términos hipótesis y condicionalidad. Es necesario llegar a los más modernos Alcina y Blecua, quienes hacen constar que «la oración introducida por si se relaciona lógicamente con la principal, de tal manera que el cumplimiento de la principal depende del cumplimiento de la subordinada de manera necesaria». Y precisan: «La base de esta relación lógica puede ser la relación de causa o efecto o, simplemente, la coincidencia lógica e interdependiente de una circunstancia de tiempo, modo, acción concomitante, etc.» ${ }^{8}$.

Resulta forzoso dejar a un lado las gramáticas y echar mano de estudios más especializados para obtener una idea cabal, en cuanto a contenido y deno-

${ }^{4}$ Rafael SECo incluye las oraciones condicionales en lo que él llama «oraciones de causalidad». Más adelante dedicaremos un espacio a tratar este asunto. Puede verse su Manual de Gramática española, Madrid, Aguilar, 1." reimpr., 1989, p. 245.

5 Véase la noción aportada por Francisco Marcos Marín en su Aproximación a la gramática española, 1." ed., 1972; 4." edición, por la que citamos, 1986, p. 270.

6 Samuel Gill GaYa, Curso Superior de sintaxis española, Barcelona, Vox, 1970, p. 318.

7 Marcos Marín dice así: «Suprimo ahora los términos prótasis y apódosis que se prestan a confusión. Prótasis es "primera parte del periodo", por lo tanto en hazlo, si quieres, la prótasis es hazlo. La apódosis es la "segunda parte del período"; en si quieres, hazlo, hazlo es la apódosis". $\mathrm{Y}$ concluye afirmando que emplear estos términos «sería un artificio sin base alguna». Cfr. op., cit., p. 271.

8 Juan Alcina y José Manuel Blecua, Gramática española, Barcelona Ariel, 1975, 1.' reimpr., 1979, p. 1132. 
minación, en el tema que nos ocupa. Lidia Conteras ha analizado en profundidad algunas de las cuestiones que aquí presentamos, y sobre los términos tan utilizados hasta entonces para los miembros de la oración condicional, dice así: «Preferimos hablar de condicionante y condicionado en vez de "prótasis" y "apódosis", respectivamente, pues los últimos términos hacen pensar que el condicionante precede siempre al condicionado, lo que no es efectivo»" ${ }^{9}$. Fue José Polo ${ }^{10}$ el siguiente en aceptar ya de entrada los nuevos términos propuestos por la lingüista chilena, con la particularidad, a mayor abundamiento, de considerarlos sintagmas. Así, llega a afirmar que la oración condicional se compone funcionalmente de dos sintagmas llamados condicionante y condicionado, de los cuales, y con independencia de la lógica filosófica, el primero es el subordinado formal y semántico del condicionado ${ }^{11}$. Con arreglo a esto, ofrecemos en esquema y por medio de un ejemplo la caracterización gramatical de las condicionales según Polo:

\section{Oración condicional}

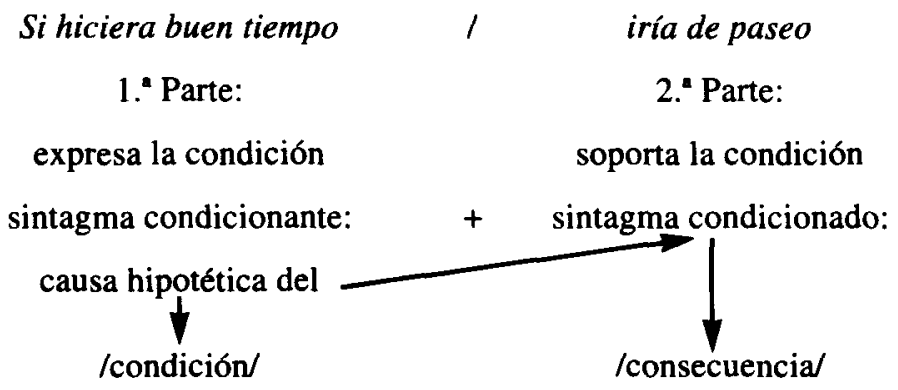

9 Lidia CONTRERAS, «Las oraciones condicionales», Boletín de Filología, Universidad de Santiago de Chile, XV, 1963, nota 10, p. 39.

10 José Polo, Las oraciones condicionales en español (Ensayo de teoría gramatical), Universidad de Granada, CSIC, 1971.

11 La lógica filosófica afecta al orden de los elementos de la oración condicional, debiendo ir siempre en primer lugar el condicionante, ya que de él depende lo que se expresa a continuación, es decir, el condicionado. De este modo, la lógica admitiría «si quieres, hazlo» pero no «hazlo, si quieres» (véase la nota 7 de este artículo). También tiene que ver la lógica con la subordinación semántica del condicionante al condicionado, a pesar de ir éste colocado el primero, ya que, como afirma Coseriu, klos esquemas formales están determinados por el significado, y no viceversa» A este respecto, puede verse el interesantísimo ensayo de Eugenio Coseriu «Logicismo y antilogicismo en la gramática», recogido en Teoria del lenguaje y lingüistica general, Madrid, Gredos, 1978, pp. (235-260), p. 249 (pp. 235-260). No podemos, por tanto, separar tajantemente forma y sustancia, porque lo formal se comprueba en la sustancia. 
Interesa que nos detengamos en este punto y recurrir a la frase latina «posita conditione ponitur conditionatum; sublata conditione tollitur conditionatum», para evitar posibles errores debidos a la igualdad de significado que algunos gramáticos han señalado para las nociones de condición e hipótesis. Desde el momento en que, si nos atenemos al arquetipo latino recién visto, tras la condición se coloca lo condicionado y éste se suprime, incluso, en aras de la condición, podemos afirmar, sin lugar a dudas, que la condición nunca puede ser posterior a la consecuencia. De ahí que la terminología etimológica prótasis-apódosis resulte precaria. Pero hay más todavía: hipótesis y condición no son lo mismo. Hipól sis, en palabras de Hoffmeister, es "toda suposición o aceptación de los fundamentos, orígenes, fuerzas, leyes y relaciones mediante los cuales se puede resolver conceptualmente un problema o rellenar un vacio de nuestra experiencia» ${ }^{12}$. En cambio, condición según Lidia Contreras, es «aquello de lo que algo depende, a lo que algo está ligado, en tal forma que esto no tiene lugar si no ocurre aquello» ${ }^{13}$. Se deduce de esto, de forma natural, que la hipótesis es más amplia que la condición y más especulativa, ya que aquélla es una suposición, algo de tipo conjetural y sin comprobación manifiesta, mientras que la condición es algo necesario, un hecho que implica la necesidad irremediable de que algo se realice a partir de un hecho, aunque éste se halle sometido al cumplimiento de un requisito. En otras palabras, la hipoteticidad no sale del ámbito de la suposición, actúa sobre supuestos, sobre una simple verdad de hecho, frente a la comprobación, que surge de la obligatoriedad de la necesidad en la condición, y que conlleva, en ocasiones, una verdad manifiesta. Esto es lo que diferencia las oraciones «Si la temperatura sube, el metal se derretirá", donde se afirma la relación del derretimiento del metal con la subida de la temperatura, de «Si hace buen tiempo, vamos de paseo", donde no está asegurado que haga buen tiempo y, en consecuencia, que vayamos a salir. Ateniéndonos a lo expuesto, estamos de acuerdo con Lidia Contreras en que existen hipotéticas que son también condicionales e hipotéticas no-condicionales. En las primeras no hay condición sin condicionado, no se da nunca antecedente sin consecuente, tienen carácter implicativo de verdad y forman un juicio complejo: «Si Dios es justo, castigará a los culpables». Por contraposición, en las segundas el carácter implicativo no está en

12 Johannes HoFfMEISTER, Wörterbuch der Philosophischen Begriff, Hamburg, F. Meiner, 2." edición, 1955, bajo «Hypothese»: «die Voraussetzung, die Annahme von Gründen, Ursache, Kräften, Gesetzen, Beziehungen, die zur Lösung von Problemen, zur Ausfüllung von Lücken der Erfahrung, zur Herstellung von Zusammenhängen, zum Begriflichmachen von Regelmässingkeiten dientm.

13 Lidia Contreras, op. cit., p. 35. 
los términos, sino en los hechos, y enuncian un juicio simple: Si se pone un fósforo encendido en la pólvora, ésta explota.

Para nosotros, en suma, la oración específicamente condicional se fundamenta en la necesidad causal, en la que produce su efecto con dependencia de otra, mientras que la hipótesis descansa sobre una necesidad meramente l6gica: que el sol amanezca mañana es algo esperado por lo habitual, pero no deja de ser una hipótesis, no tenemos seguridad absoluta de que suceda realmente ${ }^{14}$.

\section{Elementos MoRfosintácticos de LA CONDICIONALIDAD}

La oración condicional va precedida de partículas simples, compuestas y sintagmas enteros que la introducen ${ }^{15}$. La conjunción por excelencia es si. Según Bello, "el si condicional es siempre un adverbio relativo equivalente a la expresión supuesto que o dado que, tomada en el sentido de condición»; «El $s i$, adverbio condicional, lleva casi siempre envuelto su antecedente, que por tanto existe sólo en el entendimiento y pudiera representarse por el adverbio demostrativo así: «Te perdonaré si te enmiendas» equivale a «te perdonaré así, de este modo, con esta condición si te enmiendas» ${ }^{16}$.

Además del adverbio si existen otras conjunciones o adverbios con valor condicional, de los que aquí extractaremos sólo algunos: cuando, cuando no, donde, donde no, siempre que, siempre y cuando, como que, con tal que, con sólo que, conque, caso de que, ya que, a condición de que, supuesto que, dado caso que, a no ser que, y relativos de valor hipotético ${ }^{17}$.

14 Para estas cuestiones de lógica, puede consultarse el Tractatus logico-philosophicus de Ludwig Wittgenstein, Madrid, Alianza, 1973.

15 Estamos de acuerdo con José Mondéjar en que el elemento de relación o partícula marca la clase, pero nunca la modalidad de la oración condicional. A este respecto puede verse «La expresión de la condicionalidad en español», Revista de Filología Española, Madrid, XLIX, 1966, pp. 229-254, p. 235 y ss.

16 A. Bello, op., cit., (edición de Ramón Trujillo), t. I, pp. 342-344. No obstante, para otros estudiosos, como es el caso de Antonio Tovar, la partícula si comienza por significar «así, en tal caso». Cfr. A. Tovar, Gramática histórica latina. Sintaxis, Madrid, S. Aguirre, p. 213.

17 Conviene notar que las partículas cuando y siempre que son temporales cuando van con indicativo. En caso de que vayan con subjuntivo son condicionales, al igual que a condición de que, con tal que y otras recién vistas. 
Los anunciadores compuestos suelen ser sintagmas relacionantes y pronominales, del tipo: si están las cosas así, estando así, en esa situación, en tal caso, de este modo, así, etc.

Otros anunciadores sintagmáticos son formas impersonales del verbo, como infinitivo con $a$ o de, gerundio, participio y estructuras como a decir verdad, a ser verdad, de ser verdad ${ }^{18}$.

2.1. Las condicionales formuladas en infinitivo suelen ir precedidas de las preposiciones $a$, de o con: "Respondióle que luego firmara la sentencia a su favor, a tener plumas» (si tuviera plumas); «Redimen esta civilidad del gusto los sabios con hacer reflexiones nuevas...» (si hacen $)^{19}$.

2.2. Las condicionales expresadas con gerundio pueden aparecer con los verbos ser o estar: «...porque siendo la tradición común ser dos los caminos... halló con no poca admiración que eran tres...»; «Sepamos por qué los ojos y la boca han de llevar esta ventaja a los oídos, y más estando tan expuestos al engaño»; con un verbo cualquiera: «Porque ¿quién, sabiéndolo, quisiera meter el pie en un reino mentido...» (si lo supiera); «Despreciando cuanto hay, seréis señor de todo"; o bien con la preposición de ante ellos formando una construcción más arcaica «...que a ti te sucede lo que a todos los otros, que en viéndolo una vez tienen harto» (si lo ven, con verlo sólo una vez...).

2.3. Las condicionales anunciadas con participio admiten adverbios $u$ otra clase de determinantes: «Y bien examinados, no son otro que una confitada inmundicia de vicios y pecados» (y si los examinamos bien); «Subamos a aquella eminencia, que levantados de tierra yo sé que descubriremos mucho".

18 Estructuras de este tipo pueden darse con otros verbos (de saberlo antes, no hubiera venido: a poderlo evitar, lo habría evitado), ya que son estructuras condicionales del tipo de, a más infinitivo.

19 Los ejemplos que presentamos a consideración en este artículo están entresacados de la Primera Parte de El Criticón de Baltasar Gracián (pp. 103-404), edición de Miguel Romera-Navarro, Philadelphia, University of Pennsylvania Press, London: Humphrey Milford: Oxford University Press, tres tomos, 1938, 1939 y 1940, pues nos pareció interesante estudiar el funcionamiento de las oraciones condicionales en la obra maestra de un clásico de la Literatura española del siglo XVII. 


\section{Clasificación de las ORaciones Condicionales}

Entramos ahora en el aspecto más atrayente del tema de nuestro estudio, ya que la condicionalidad puede venir expresada, no sólo por los elementos relacionantes expuestos en el apartado anterior, sino también por otros que introducen, aparentemente, oraciones de tipo temporal, causal, consesivo, etc. Ello ocasiona lugares de coincidencia entre las múltiples clases de proposiciones adverbiales, generados por los dos planos que manifiesta la condicionalidad, el formal - si nos atenemos a la partícula introductoria - y el semántico, emanado del significado intrínseco de la condicional. Dicho de otro modo, estimamos imprescindible dejar muy claro que una cosa es la construcción formal de una oración $\mathrm{X}$ y otra muy distinta el contenido de esas construcciones que «parece» indicar un tipo de proposición cuando, en realidad, se trata de otro diferente.

En este apartado examinaremos dos cuestiones de importancia: el ordenamiento de las condicionales por sus cruces con otras adverbiales y según los distintos empleos de los tiempos verbales.

\subsection{Cruces y matices de las oraciones condicionales}

Los matices o cruzamientos que ofrecen interés y tienen carácter real son siete: el causal, concesivo, final, consecutivo, comparativo, temporal y enunciativo $^{20}$.

20 Discrepamos de Polo en la inclusión que lleva a cabo de los matices dubitativo, locativo y enunciativo como oraciones condicionales en cuanto a la forma (Véase J. POLO, op. cit., $*$ La condicionalidad y su delimitación de otros matices», pp. 47-121, y más concretamente pp. 73, 78 y 80). En nuestra opinión, en las adversativas no existe condición. Polo aduce que en ellas la condición deja de funcionar para derivar en una oposición, pero esto es falso: la contrariedad no tiene nada que ver con la condicionalidad, al menos tal y como creemos que debe ser entendida. En cuanto a las dubitativas, que son para Polo variantes de inseguridad o hipoteticidad, tampoco son condicionales por la falta, nada menos, de la consecuencia. Como ya vimos anteriormente (apartado 2, de este artículo), no toda hipótesis es condición; para que haya condición es necesario que de la hipótesis dependa la efectividad de una consecuencia. El matiz locativo, fácilmente identificable con el temporal, tampoco se neutraliza en condición. Y finalmente, las explicativas son, en realidad, dubitativas, porque carecen de consecuencia. La conjunción subordinativa si en esta clase de oraciones suele introducir la subordinada sustantiva de un verbo de duda, interrogación y semejantes. 
Para concluir, estudiaremos las llamadas intercondicionales.

3.1.1. Las condicionales son oraciones muy próximas a las causales. Así lo han visto algunos filósofos y lingüistas. Reconocemos con Cejador ${ }^{21}$ que «las proposiciones condicionales pertenecen a las causales, ya que la condición influye, aunque no sea más que extrínsecamente, en la realización del hecho». Es más, la condición expresa una causa lógica, pero es hipotética. Rafael $\operatorname{Seco}^{22}$ nota asimismo el matiz causal de las oraciones condicionales y, como ya vimos anteriormente, recalca: «Quedan incluidas en este grupo las condicionales y concesivas que guardan con sus oraciones subordinantes un lazo de causalidad, toda vez que la condición es antecedente indispensable para un cierto efecto, y en lo concesivo hay un obstáculo previo, especie de condición, aunque desdeñable».

Abbagnano $^{23}$ se refiere a la condición en estos términos: «Por lo general es lo que hace la previsión probable de un acaecimiento». Sin embargo estamos de acuerdo con Ferrater ${ }^{24}$ al asegurar que existe una diferencia entre causa y condición, pues mientras la primera «es positiva: aquello por lo que algo es o sucede, la condición tiene un sentido negativo: aquello sin lo cual algo no sería o sucedería». Veamos estos ejemplos: «Como lo rompiste, te castigo» (es decir, te castigo por haberlo roto, porque lo has roto), y su diferencia con el que sigue: "Como lo rompas, te quedas castigado» (es decir, si lo rompes, en el supuesto caso que lo rompas...). Aquí la negatividad está en que si no lo rompe no sufrirá el castigo. O el introducido por si: «Verdad será si tú lo dices (= porque tú lo dices). Debemos tener en cuenta, no obstante, que son dos planteamientos distintos, dos maneras diferentes de establecer la relación entre los términos, ya que la causa propiamente dicha (primer ejemplo) es suficiente por sí misma, mientras que la condición es necesaria, pero al fin hipotética. Es una hipótesis causal: algo posible que actúa como verdadera causa o algo imposible que, si llega a ser real, también se conduciría como causa. En suma, las oraciones condicionales son una categoría de las causales, como si se hubieran desprendido de ellas, permaneciendo unidas por un nexo en común: la causalidad.

Los casos que ofrecemos ahora a consideración están introducidos por auténticos nexos causales: pues, ya que, que..., fácilmente sustituibles por un

21 CEJADOR, op. cit., p. 475.

22 R. SECO, op. cit., bajo «Oraciones de causalidad», p. 245.

23 Nicolás Abbagnano, Diccionario de Filosofia, Buenos Aires, 1977, bajo «Condición».

24 José FerRater MORA, Diccionario de Filosofía, Madrid, Alianza Editorial, 4 vols., 1986, vol. I; bajo *Condición». 
si al menos en su relación lógica, totalmente hipotética: «Aun creería que soy algo, pues así me persigues...» (si me persigues de ese modo, podría creer incluso que soy algo importante); «...pero ya que hayan de ser dos no más [los ojos] pudiéranse repartir y que uno estuviera delante para ver lo que viene...» (si han de ser dos solamente, pudiéranse o se podrían repartir).

3.1.2. La concesión supone un paso adelante en la relación de causalidad, ya que la condición entraña relación inevitable entre dos hechos y la concesión afirma el hecho a pesar de todo. Ya Meyer-Lübke ${ }^{25}$ habló de la cercana afinidad condicional-concesiva. Cejador ${ }^{26}$ llega a afirmar incluso que «las proposiciones condicionales muchas veces no lo son más que en apariencia, siendo en realidad concesivas». Alonso del Río ${ }^{27}$ estima que si prescindimos de lo condicional por ineficaz, acabaremos en las concesivas. Y propone el ejemplo: «Si le acometieran todos los demonios del mundo, no volviera pie atrás».

El carácter concesivo de las proposiciones condicionales se demuestra cambiándolas por adversativas: «...Alargó sus brazos para recogerle en ellos, amarras de un secreto imán, si no de hierro..." (aunque, pero no de hierro); «Ya que les faltó el conocimiento, no el presagio de sus males, y si no los concibe, los adivina" (y aunque no los concibe). Incluso hemos encontrado un caso donde el carácter concesivo se refuerza con un aunque: «... Pues si aunque todos los entendimientos de los hombres que ha habido ni habra se juntaran antes a trazar esta gran máquina del mundo... jamás pudieran atinar a disponerla». Está claro que si suprimimos el aunque, la frase sigue siendo concesiva.

3.1.3. La finalidad coincide con la pura causalidad: es lo que se ha llamado causa final o fin causal: "Te regalo esto porque te portes bien" (porque y para que te portes bien). De otro modo, la finalidad por la que algo se realiza constituye un hecho, una verdadera causa, y puesto que la condición tiene que

25 Wilhelm MEYER-LUBKE, Grammaires des langues romanes, Paris, 1923, t. III, p. 179.

26 CEJADOR, op. cit., p. 488.

27 Alonso del Rfo en su Gramática Española, Madrid, 1973, p. 294, al hablar de la proximidad condicional-consesiva, dice que «la concesión rompe la causalidad hipotética para mostrar que la causa existem, pero que no la juzga «capaz de impedir la realización o irrealización del acton. A nuestro juicio, se trata de una causalidad muy especial, sin duda, porque no es causa que origine un efecto positivo, sino negativo: un obstáculo, en suma. 
ver con la causalidad, la finalidad también. Se podría decir que la causa es una finalidad inicial, y el fin, terminal. La relación entre ambas es la de ser dos presupuestos, aunque diversos: la dirección o finalidad es un punto de llegada, algo que se plantea y la condición, punto de partida, es un presupuesto que condiciona o predetermina, como condiciona, de alguna manera, la finalidad: «¿Quién me ha dado el ser y para qué me lo ha dado? Para estar aqui metido, grande infelicidad sería» (es decir, gran infelicidad sería si se tratara de estar aquí metido) ${ }^{28}$.

3.1.4. Otro aspecto de la causalidad lo constituye la oración consecutiva: «No voy porque ha llovido mucho» equivale a «ha llovido mucho; por lo tanto, no voy» y a «ha llovido tanto que no voy». La consecutiva expresa causalidad entre el hecho y la consecuencia. Con un mismo ejemplo para los tres tipos de oración podemos observar cómo la dirección en las causales y condicionales es común (un «hacia»), siendo contraria en las consecutivas (un «después»):

O. Causal:

O. condicional:

O. consecutiva:
No salí

Efecto

Si llueve

Condición

(o causa)

Llueve mucho porque llovía mucho Causa

no salgo

Hecho

(o consecuencia)

no salgo, por tanto

\section{Causa $\longrightarrow$ Consecuencia}

«...Que a veces llegan tan a los extremos los males que pasan a ser dichas". Causa: se extreman los males... Consecuencia: llegan a ser dichas... "No está aquí el verdadero bien, éste es el mal de los males: luego errado habemos el camino». Causa: no está aquí el bien verdadero. Consecuencia: nos hemos equivocado.

28 En esta oración, el carácter condicional proviene, en realidad, del hipotético sería. Acerca del valor que confieren a las condicionales los tiempos verbales, hablaremos más adelante en el epigrafe 3.2. de este artículo. 
3.1.5. Según Tovar, el primer valor de la partícula si es el comparativo ${ }^{29}$. Lo mismo opina Lenz: «la conjunción condicional si del latín es la misma palabra que, con añadidura de la partícula demostrativa ce, da sic, es decir, si significa propiamente "asi", de modo que el período hipotético consta de dos oraciones coordinadas que contienen una comparación: "Si vienes, te daré pan" es primitivamente "así vienes, (asî) te daré pan"»" «Así vienes, así te invito» son dos sintagmas correlativos de comparación, dependiendo la realización de uno de ellos de la realización del otro. Son dos sintagmas en los umbrales de la condicionalidad.

No cabe duda, pues, que el período hipotético contiene, asimismo, una comparación, dado que los términos expresan, una vez más, causalidad, por la dependencia y correlación entre ellos. Pero es necesario precisar que una cosa es que una relación formulada mediante estructuras gramaticales comparativas pueda formularse también mediante una estructura condicional, y otra muy distinta el que tal comparación tenga carácter condicional, cuando, además, en muchos casos no son equivalentes. De aquí se desprende el carácter condicional de este caso: «Cuan gustosa ha sido para mí tu relación, tan penosa ha de ser la mía» (si gustosa ha sido tu relación...), pero no el de este otro exclusivamente comparativo: «Así como el sol es claro espejo de Dios y de sus divinos atributos, la luna lo es del hombre».

3.1.6. Igual que con las comparativas, sucede con la coincidencia tempo$\mathrm{ral}$, no equiparable a condicional cuando se trate de dos formulaciones diferentes, correspondientes a dos estructuras mentales distintas. Bajo una formulación temporal, la relación sólo está pensada como temporal en estos dos casos, introducidos por el adverbio cuando intercambiable por si, siempre que el modo verbal sea el subjuntivo: «Y dime Egenio, amigo, cuando le hallásemos hecho un bruto, ¿cómo le podríamos restituir a su primer ser de hombre?» (y si le hallásemos hecho un bruto...); «Cuando vieres un presumido de sabio, cree que es un necio..." (si vieres un presumido...), pero nunca en «Cuando habia de correr, la detiene, y cuando había de ir con tiento, vuela», claramente temporal.

29 Véase la nota 16 de este artículo.

30 Rodolfo LENz, La oración y sus partes, Madrid, Centro de Estudios históricos, 1920, p 433. También en Revista de Filología Española, 1935. 
3.1.7. En el caso del matiz enunciativo, éste enuncia a través de lo condicional, para que, establecida la relación de causalidad, la aserción resulte más convincente. Bello ${ }^{31}$ la llamó con acierto «hipótesis formal o gramatical»: «Si lo que se vende es callar, ¿la paga cómo ha de ser callar?» Es admisible que el segundo elemento sea una oración exclamativa o interrogativa: «... que si la sombra es tal, ¡cuál será su causa y realidad a quien sigue!»; «Pues si esto es verdad, ¿qué quedará para la mentira?».

3.1.8. Las oraciones intercondicionales van introducidas por las partículas como si, por si. Como si revela una comparación modal supeditada al cumplimiento de una condición. Lidia Contreras ${ }^{32}$ cita a Hanssen, para quien son «fragmentos de cláusula hipotética»; y a A. Alonso: «"como si" más subjuntivo encierra una comparación hipotética, con frecuencia de negación implícita, en la cual "como" es adverbio comparativo». Rafael $\mathrm{Seco}^{33}$ las denomina «comparativas irreales». En opinión de Salvador Fernández ${ }^{34}$, estas oraciones suponen «un verdadero empleo metafórico, ya que toda comparación es principio de metáfora, e incluso puede haber un uso alegóricon.

En realidad, las modales comparativas representan un paso hacia o desde la fusión o metáfora: «es como si fuese un mar, es como un mar, es un man". En El Criticón se da un uso metafórico y otro alegórico: «Andrenio, al hacer un grande extremo, alzó los ojos y gritó al cielo, como si le hicieran ver las estrellas» ("Ver las estrellas» = «sentir dolor intenso», es metáfora lexicalizada); «Estaba fabricada de unas piedras tan atractivas que atraían a sí las manos y los pies, los ojos, las lenguas y los corazones como si fueran de hierro..." (Alegoría: piedras tan atractivas [como el imán] que atraen ojos, lenguas y corazones como si fueran de hierro).

En el caso de intercondicionales introducidas con por si, los gramáticos se plantean si tienen matiz condicional, causal o final. Afirmamos con José Polo $^{35}$ que «se trata de un período causal hipotético con una idea de finalidad implícita, y no de un período condicional». Lidia Contreras ${ }^{36}$ analiza de modo

31 BeLlo, op. cit., p. 113.

32 Lidia Contreras ha estudiado las diferentes construcciones intercondicionales en dos artículos: «El período comparativo hipotético con "si"», Boletín de Filologia, Universidad Santiago de Chile, X, 1958, pp. 39-49 (La cita es de la página 40), y en “El período causal hipotético con "si"», Boletín de Filología, Universidad de Santiago de Chile, XI, 1959, pp. 353-359.

33 R. SeCO, op. cit., pp. 353-359.

34 Salvador Fernández RAMtrez, "Como si + subjuntivo», Revista de Filología Española. Madrid, XXIV, 1937, pp. 372-380, p. 372.

35 J. POLO, op. cit., p. 117.

36 L. CONTRERAS, «El período causal hipotético con "si"», p. 357. 
diferente el ejemplo de la Academia: «te lo digo POR SI no lo sabes = te lo digo por enterarte (para que te enteres o lo sepas) si no lo sabes», como: «te lo digo por si no lo sabes (porque pudiera ser que hasta ahora no lo supieras) [para que lo sepas])». De este modo, la proposición encabezada con «por sì) muestra un hecho supuesto por el interlocutor, que actúa como una causa de la acción expresada en la proposición anterior, permaneciendo inherente en ella la idea de finalidad: «Preguntó si estaría a mano la llave $=$ Sí - dijo uno- yo la tengo encomendada por si llegan a verla». También se da el caso de reforzamiento del si con el verbo ver. De este modo, el giro «por ver si» o «para ver si» implica mayor intencionalidad en el propósito: «Duplicábame, aun no bien singular, por ver si (por si) apartado de mi ignorancia, podría dar alcance a mis deseos»; «Argüíame tal vez, para ver si empeñado me excedería a mí mismo».

\subsection{Usos de los tiempos verbales en la oración condicional}

Entramos en una de las cuestiones más problemáticas de las oraciones condicionales. Gili Gaya ${ }^{37}$ las divide, según que la prótasis ${ }^{38}$ vaya en indicativo o subjuntivo, en reales e irreales, aunque sin olvidar el ser hipotético o eventual de toda oración condicional. Lidia Contreras ${ }^{39}$ se decide, entre un conjunto extenso de posibilidades, por la dicotomía posible-imposible que, a nuestro entender, equivale a la división necesaria-imposible de la Academia ${ }^{40}$. El tercer modo de esta relación -el contingente- es un matiz de lo posible. Este último régimen las clasifica en seguras, probables e improbables.

A nuestro juicio, la denominación posible-imposible quizá resulte más clara que la de real-irreal ${ }^{41}$. Por ello suscribimos la clasificación que lleva a cabo Rafael Lapesa para el español antiguo:

37 GILI GAYA, op. cit., p. 319.

38 La mayor parte de los gramáticos, salvo Lidia CONTRERAS y algún otro aislado, utilizan constantemente la terminología prótasis/hipótesis. Nosotros, vista la insuficiencia de dichos términos, optamos por la más clara de condición y consecuencia, para hipótesis y prótesis respectivamente. Véase además la nota 7 de este mismo artículo.

${ }^{39}$ Lidia CONTRERAs, «Las oraciones condicionales»..., p. 46.

40 Esbozo..., pp. 458 y 433.

41 Algo es real cuando es posible, cuando se puede concretar (plano de la esencia), siendo lo posible lo que se abstrae de lo real, frente a lo imposible, que es de todo punto irrealizable (plano de la experiencia). 
3.2.1. Hipótesis ${ }^{42}$ real o posible: Condición en indicativo y consecuencia en indicativo, o bien tiempo y modo libres. Ello equivale a la noción de necesaria que admite la Academia, donde, puesta la condición, se afirma lo condicionado como cierto: «Si vuelves, te espero».

3.2.2. Hipótesis irreal o imposible: La consecuencia expone un hecho que no es real y, por eso, al no verificarse en la condición, afirmamos implícitamente que no es posible: «Si hubieras contestado, te habrian aprobado». La condición puede ir en imperfecto de subjuntivo -se o en pluscuamperfecto de subjuntivo - ra o -se; la consecuencia, en condicional, imperfecto o pluscuamperfecto de subjuntivo.

3.2.3. Hipótesis contingente: ni se afirma ni se niega la relación entre condición y consecuencia, por tratarse de algo hipotético: «Si me convidas a comer, te acompañaria». Se distinguen en esta categoría la hipótesis posible y la dudosa, a las que llamaremos probable e improbable respectivamente:

a) Probable: Condición en futuro hipotético y consecuencia en futuro de indicativo, o tiempo libre.

b) Improbable: Condición con subjuntivo -ra o -se y consecuencia con potencial.

\subsubsection{Hipótesis real o posible: condición en modo indicativo}

Cuando la condición está en indicativo, no pueden figurar en ella los tiempos futuros: *si vendrá, si habrá venido se sustituyen por «si viene o si ha venido"; ni los condicionales simple o perfecto: *si vendría o si habría venido se sustituyen por «si viniera o viniese o si hubiera o hubiese venido». Tampoco admite la condición el pretérito anterior, pero el resto de los tiempos de indicativo caben en ella.

La consecuencia admite el imperativo, cualquier tiempo del indicativo menos el pretérito anterior, y cualquiera de subjuntivo menos los futuros.

42 Rafael LAPESA, apuntes orales del Seminario de Doctorado «Morfosintaxis histórica del verbo español», 1973, bajo «El período hipotético en español antiguo». 


\subsubsection{Presente en la condición y presente en la consecuencia}

El presente, tiempo imperfecto, ve la acción en su transcurso y coincidente con el momento en que hablamos. Además, como tiempo relativo, se tiñe de un significado futuro: «...que si los hombres no son fieras es porque son más fieros» (será porque son más fieros, con ajuste perfecto del futuro).

El presente se emplea, sobre todo, cuando lo que se plantea como condición es una premisa, de la que se infiere una consecuencia lógica. En casos como éste, el sentido condicional de si es aparente, porque expresa una verdad manifiesta que asevera con más fuerza: «Si hay ley, si hay razón, si justicia en el mundo, la grandeza de los beneficios bastaría...». Es el caso de juicios o afirmaciones categóricas, extraídos de la condición como algo natural: «...pues cada uno [cada hombre] es un lobo para el otro, si ya no es peor el ser hombre" (el hombre es lobo para el hombre es lo que se afirma aquí, calcado de la frase latina: «homo homini lupus est»).

\subsubsection{Presente + Futuro $^{43}$}

Como ya vimos en 3.2.1, el futuro no puede figurar en la condición, pero sí en la consecuencia ${ }^{44}$. La razón de esta incompatibilidad está en la incertidumbre de la acción futura por su índole venidera, que se suma a la hipoteticidad de las condicionales y entra en conflicto con la seguridad expresada por el futuro absoluto. Casos de este tipo son abundantes y aparecen reflejados en cualquier modalidad estilística: «Mas si quieres saber el material suceso de mi vida, yo te lo referiré...»; «... y con la misma facilidad te desataré a ti, si quieres»; «Sepamos el precio... que no sé si lo hallaremos en otra parte». Incluso se da con oración interrogativa: «Pues si esto es verdad, ¿qué quedará para la mentira?».

43 A partir de aquí y para las siguientes enumeraciones, el verbo expresado en primer lugar corresponde a la condición y el segundo a la consecuencia (véase el reciente apartado 1).

44 El empleo obligatorio del presente de indicativo después de si data de la época en que el latín vulgar reemplazó el futuro por el presente. Pero conviene añadir que ya en latín el futuro no es frecuente en la hipótesis, y que el nuevo futuro castellano con su idea obligativa se prestaba bien para la apódosis, pero debía de repugnar para la hipótesis. Por eso «Si vienes te doy pan» pasó a «Si vienes, te daré pan». 


\subsubsection{Presente + Potencial}

Las denominaciones de «futuro hipotético» según Gili Gaya ${ }^{45}$ y de «pospretérito» por parte de Bello ${ }^{46}$ para el potencial o condicional apuntan a la noción que da la Academia para este tiempo: «expresa una acción futura en relación con el pasado que le sirve de punto de partida ${ }^{47}$. Sin embargo, otros autores lo consideran «imperfecto del futuro», lo cual es también verdad, ya que su carácter imperfectivo deja indeterminado el desenlace de la acción, la cual puede ser presente, pasada o futura: "Que si un buen consejo es bastante para hacer dichosa toda la vida, iqué obrarían en él tantos y tan importantes?» (acción del potencial coincidente con el presente de la condición); «Mas si ahora nos admira un diamante por lo extraordinario, una perla peregrina, ¿qué ventaja sería en Andrenio llegar a ver de improviso un lucero, un astro, la luna, el sol mismo...» (acción del potencial anterior con respecto al presente); "Que si los hombres son otros de la noche a la mañana, iqué sería en aquel centro de la mentira!» (acción del potencial futura en relación al presente de la condición).

\subsubsection{Presente + Imperativo}

El imperativo, tiempo independiente, es necesariamente futuro: «Con todo, si queréis ver alguno, buscad un cardenal Sandoval en Toledo, un conde de Lemos...». También es un claro equivalente del subjuntivo en oraciones negativas o impersonales: «No te apartes un punto de mi lado, si no quieres perderte»; «Si tales disparates hay en el mundo, llámese casa de orates hermanados».

\subsubsection{Presente + Imperfecto de subjuntivo}

Los tiempos del indicativo expresan la realidad; los del subjuntivo la irrealidad. Si nos atenemos a esta regla fija, el caso presente tendría valor

45 Gill Gaya, op. cit., p. 167.

46 BELLO, op. cit., t. I, p. 434.

47 Esbozo..., p. 472. 
irreal por tratarse de un imperfecto de subjuntivo. Pero ello no es totalmente cierto, ya que Lenz ${ }^{48}$ advierte sobre la procedencia de cantara, que es precisamente cantaveram y, en consecuencia, sinónimo de «había cantado». No puede ser olvidado, por tanto, junto al valor de subjuntivo, el antiguo e histórico valor de indicativo para esta forma: «Pues si el preguntar comienza en el ignorar, mal pudiera yo responderle», claro equivalente de «mal podría yo responderle».

\subsubsection{Imperfecto + Imperfecto}

El imperfecto expresa acción pasada, que sólo interesa en su duración. Su carácter inconcluso le confiere aspecto de mayor duración que los demás pretéritos: «Decía que era otra Circe, si no (era) peor cuanto más encubierta con capa de hacer bien». Sin embargo, en las oraciones condicionales se emplea este tiempo con significado futuro sustituyendo a la forma -ria de la consecuencia y aun a las formas - ra y -se en la condición: «... y añadieron que en pago de tanto beneficio le avisaban [avisarían] huyese luego, antes que el hombre saliese, si no quería perecer allí a manos de su fiereza». La sustitución por la forma -ra es clara, aunque el verbo se encuentre elidido en el texto: «Calidades (eran) todas muy a cuento, si no (eran) (fueran) muy a propósito para mozo de ciego».

\subsubsection{Imperfecto + Futuro perfecto}

El futuro perfecto, llamado «antefuturo» por Gili Gaya ${ }^{49}$, expresa acción venidera anterior a otra también venidera: «Cuando lleguéis habremos cenado" muestra el acabamiento de la cena con relación a la llegada de nuevas personas. El caso recogido en $E l$ Criticón responde a este esquema, aun-

48 LENZ, op. cit., p. 464.

49 GilJ GAYA, op. cit., p. 166. 
que presenta matiz causal: «Si era entendido, como dices, ... allá [la corte de Artemia] habrá aportado» («habrá llegado, pues era entendido»).

\subsubsection{Imperfecto + Potencial}

El valor de estos tiempos ha quedado visto en los apartados 3.2.1.3 y 3.2.1.6 de 3.2.1. La acción transcurrida ya del imperfecto, con aspecto de futuro, es anterior a la hipótesis pendiente de realización por parte del potencial: «...Le dijo que aunque estaba de ronda, si era servido, le irían acompañando todos sus ministros».

\subsubsection{Pretérito + Presente}

El pretérito es tiempo perfecto y acabado, como forma absoluta del pasado. Su significación perfectiva y absoluta, sin relación alguna con el momento en que hablamos, da al pretérito sentido de negación, relativa al presente: «Pues dime, ¿con qué hacen tanto mal los hombres si no les dio la naturaleza armas como a las fieras?». Puede haber algún caso con presente de subjuntivo en la consecuencia, pero como en el ejemplo anterior, insinuar que una cosa fue es decir que ya no es: «...Que si se adelanto a ocupar una oreja la mentira, se conserve la otra intacta para la verdad».

\subsubsection{Pretérito + Pretérito}

Las acciones de ambos verbos están ya concluidas: «El vulgo de las aves le recibió con salva de armonía, si ya no fue darle la vaya».

Hemos encontrado un caso con pretérito perfecto compuesto en la consecuencia. Expresa el pasado inmediato, pero guarda relación con el presente. De ahí que Bello ${ }^{50}$ lo llamase «antepresente», si bien lo utilizamos a veces para acciones apartadas del presente: «Mucho has perdido si un amigo perdiste».

50 Bello, op. cit., p. 435. 
3.2.2. Hipótesis irreal o imposible: condición en modo subjuntivo

Cuando la condición está en subjuntivo, sólo puede llevar imperfecto (formas - ra y -se) en los tiempos presente y futuro, y pluscuamperfecto en el pasado. Entramos así en las condicionales irreales, en las que la condición es un hecho que estimamos no sucedido en el pasado, irrealizable en el presente $o$ improbable en el futuro; e igualmente en la consecuencia. Hay tres esquemas sintácticos:

Primer esquema:

Condición: Imperfecto de subjuntivo en -ra o -se.

Consecuencia: Potencial simple o presente de indicativo.

\subsubsection{Imperfecto de subjuntivo -ra + Imperfecto -ra}

La forma -ra de la condición es plenamente subjuntiva . Prueba de ello es su posible sustitución por la forma -se, de la que es su absoluto sinónimo, mera variante formal, por tanto ${ }^{51}$. El empleo de -ra en la condición es obligado, pero no así su aparición en la consecuencia que, según Seco $^{52}$ «es anticuada y sólo aparece de vez en cuando en el español literario». Es decir, que la colocación de -ra en la consecuencia conserva el valor de indicativo originario, y puede sustituirse por el condicional -ría. Resumiendo:

amara equivale a amase en la condición de las condicionales

amara equivale a amaría en la consecuencia de las condicionales

51 Amara procede del pluscuamperfecto de indicativo latino amaveram, y amase del pluscuamperfecto de subjuntivo amavissem. La identificación de significados entre amara y amase es resultado de un largo proceso histórico que los ha ido aproximando progresivamente, sin que haya llegado a ser tan completa que permita permutarlos entre sí en todos los casos. El predominio de amara con valor subjuntivo se inicia en el siglo XV y continúa el avance en los escritores del Siglo de Oro.

52 SECO, op. cit., p. 226. 
Sin embargo, en El Criticón no hemos encontrado ningún caso de potencial en la consecuencia y de - ra en la condición, prueba de que -ra prevalece en la lengua culta y literaria, y -se predomina más en el habla corriente: «...Que si no fuera con este universal ardid, ninguno quisiera entrar en un tan espantoso mundo y que pocos aceptaran la vida si tuvieran estas noticias antes»; «Esos abrojos son los que lastiman, que si tú fueras cubierto de flores, yo sé te quisieran».

3.2.2.2. Imperfecto - ra + Presente de indicativo ${ }^{53}$

«...Pero la mayor parte la hallaréis acullá sobre el cuerno de la luna, y aún pretenden subir más alto si pudieran».

Segundo esquema sintáctico:

Condición: Pluscuamperfecto de subjuntivo en sus dos formas.

Consecuencia: Pluscuamperfecto de subjuntivo -ra y potencial perfecto o simple.

a) Pluscuamperfecto -ra + Pluscuamperfecto -ra

El pretérito pluscuamperfecto de subjuntivo muestra una acción pasada respecto de otra pasada, dentro del sentido general del modo subjuntivo. Y así como en el imperfecto el uso de -ra en la consecuencia es hoy poco frecuente fuera de la lengua literaria, en el pluscuamperfecto hay empleo indistinto de hubiera o habría más participio en la lengua usual. El carácter perfectivo de hubiera señala con precisión el tiempo en que la acción se produjo y refuerza

53 El presente esquema no lo cita la Academia, pero sí se da en El Criticón. 
su duración: «...Que si él hubiera asistido al lado del divino Hacedor, muchas cosas se hubieran dispuesto de otro modo...». Hubieran dispuesto expresa un hecho pasado con relación a otro pasado: el de haber asistido. Como vemos, el uso de - ra en la consecuencia no se siente como arcaico.

b) Pluscuamperfecto -ra + Imperfecto -ra

Aquí la forma -ra es el equivalente del potencial por estar colocado en la consecuencia: «Y si esto no hubiera prevenido iqué fuera de su crueldad! Ya hubieran acabado con todo.

3.2.3. Hipótesis contingente: condición, normalmente, en futuro de subjuntivo

Tercer esquema sintáctico:

Condición: futuro simple o perfecto de subjuntivo

Consecuencia: presente o futuro imperfecto de indicativo u oración exhortativa o potencial simple

Comenzamos por un caso que tiene cabida en el segundo esquema sintáctico, pero que, a la vez, forma parte de las condicionales contingentes, que son probales e improbables.

\subsubsection{Imperfecto -se + Potencial -ría}

Ya Rufino J. Cuervo había definido bien los empleos de -ra y -se en la lengua literaria del Siglo de Oro: «En nuestros clásicos, la forma -se predomina como verdaderamente subjuntiva después de verbos que rigen este modo, 
en frases finales, optativas, adversativas, concesivas, etc., y en la hipótesis de las oraciones condicionales; la forma -ra en la apódosis y en frases que pudiéramos llamar potenciales, en las cuales se representan los hechos como meramente posibles, y que son en cierto modo oraciones incompletas por faltarles una hipótesis vaga, que varía según los casos» ${ }^{54}$. "Y muchos no sabrían, si no pensasen que saben».

\subsubsection{Futuro de subjuntivo + Futuro de indicativo}

El futuro simple de subjuntivo presenta el hecho como no acabado y siempre como contingente. Este tiempo permanece hoy en día solamente en algunas frases hechas del habla coloquial: «A donde fueres haz lo que vieres" y también en el lenguaje jurídico y arcaizante, donde se emplean todavía las llamadas condicionales contingentes, es decir, las condicionales reales de futuro con la condición en futuro de subjuntivo: "Si transcurrido el plazo no se presentare (no se hubiere presentado) perderá sus derechos».

Lenz Ilama a estas formas verbales «subjuntivo hipotético o subjuntivo hipotético de futuro" ${ }^{55}$. En el lenguaje literario y en el forense, se usa para acciones que se piensan como anteriores a un futuro que no tendrá lugar si la otra acción no se ha realizado todavía: de ahí su valor hipotético: «El que falsificare el sello del Estado e hiciere uso del sello falso, sufrirá la pena...» (ejemplo de Lenz). Falsificare e hiciere son acciones que se piensan como anteriores a sus consecuencias expresadas en futuro.

a) Futuro simple de subjuntivo + Futuro de indicativo

«Y advierte que si fuere pobre, jamás será entendido ni cortés»

54 Rufino José Cuervo, Disquisiciones sobre filología castellana, edición, prólogo y notas de Rafael Torres Quintero, Bogotá, Instituto Caro y Cuervo, 1953.

55 LENZ, op. cit., p. 465. Y añade este gramático: «No es enteramente injustificado denominar la forma -re "subjuntivo de futuro", por la incertidumbre que encierra. Aunque es mejor llamarlo "futuro hipotético" como lo hace Hanssen".

Este futuro, no obstante, está hoy en franca decadencia, pues entró desde muy pronto en rivalidad con el presente de indicativo o con el imperfecto de subjuntivo "cantase, tuviese". Era algo intermedio entre los dos. Si el lenguaje era enérgico, se prefería el presente de indicativo; si se dudaba, se prefería el segundo. Cantare tenía matiz de duda, intermedio entre ambos. 
b) Futuro simple de indicativo + Presente de subjuntivo

«Quien quisiere ver esta filosofía confirmada con la experiencia, escuche esta agradable relación».

El hecho de haber encontrado solamente estos futuros de subjuntivo nos muestra la decadencia de este tiempo en la época barroca de Gracián.

A modo de conclusión recordaremos que no toda hipótesis es condición; para que haya condición es necesario que de la hipótesis dependa la efectividad de una consecuencia. De ahí que las dubitativas, por ejemplo, subordinadas sustantivas objeto directo de verbo de duda, interrogación o equivalente, presenten un $s i$ que no es condicional: «Si vino, no lo sé», al igual que las ponderativas como «si será difícil que...», de carácter consecutivo. El hecho de que las partículas condicionales sean comunes a otras clases de oraciones hace que se vean, a veces, teñidas de otros valores. Así en «le subiré el sueldo, siempre que cumpla con sus obligaciones», siempre que equivale a «con tal que», «a condición de que». Aceptar la condicionalidad equivale, pues, a admitir el carácter hipotético ( no envidies si no conoces») y la intima conexión de causalidad existente entre condición y consecuencia, ya que sin aquélla, ésta no existiría. Además, como ya vimos, la condición nunca puede ser posterior a la consecuencia. Normalmente, lo condicionado va detrás de la condición, e incluso puede llegar a desaparecer para dar prioridad a la condición.

En otro orden de cosas, la hipótesis engloba a la condición, diferenciándole de ésta en que implica suposición, mientras que la condición expresa necesidad.

La delimitación de lo condicional se torna apasionante y, en ocasiones, difícil, ya que una oración puede ir formulada mediante una estructura condicional, o bien, formulaciones de otra índole pueden estar expresando en su estructura profunda una auténtica condición: «Si no la tenéis, causa deseo; si la tenéis, cuidado; si la perdéis, tristeza» (ejemplo de El Criticón). Aquí la formulación es de tipo temporal. Referente a la estructura de la oración condicional, ya quedó claro que una cosa es la construcción formal de una oración y otra muy distinta el contenido de esa construcción, que puede parecer indicar un tipo de proposición cuando, en realidad, se trata de otro diferente. A mayor abundamiento, y en el caso concreto de las condicionales con valor comparativo, una cosa es que una relación formulada mediante estructu- 
ras gramaticales comparativas pueda formularse también mediante una estructura condicional, y otra muy distinta el que dicha comparación tenga carácter condicional.

Pero no conviene olvidar que los tiempos verbales juegan un papel importantísimo, debiéndose a sus distintas combinaciones, las múltiples clasificaciones de oración condicional. Hay un claro predominio de los esquemas presente + presente para la hipótesis real, y de imperfecto de subjuntivo -ra + imperfecto de subjuntivo - ra para la hipótesis irreal, siendo más abundante la hipótesis real o posible. Por último, asistimos a la franca decadencia del futuro de subjuntivo para la hipótesis contingente. Ésta comienza en la edad media y de modo excesivo se da en las generaciones barrocas de Lope, Quevedo y Gracián. Sus funciones fueron absorbidas por el presente de indicativo, el de subjuntivo («cuando viniere pasó a cuando venga»), y también por las formas en -ra o-se. 